\title{
Application of Business Intelligence using Machine Learning Approach
}

\author{
Zainab Pirani, $\mathrm{PhD}$ \\ Computer Eng \\ Dept.,MHSSCOE \\ Mumbai, India
}

\author{
Anuja Marewar \\ Computer Eng \\ Dept.,MHSSCOE \\ Mumbai, India
}

\author{
Zainab Bhavnagarwala \\ Computer Eng \\ Dept.,MHSSCOE \\ Mumbai, India
}

\author{
Madhuri Kamble \\ Computer Eng \\ Dept.,MHSSCOE \\ Mumbai, India
}

\begin{abstract}
As the online marketing businesses are expanding it is difficult for e-commerce organizations to keep a track of the products in its inventory[1]. The manual process is a slow process and each and every product needs to be scanned, recorded and then be stored. This probably leads to wasting much of their time switching between applications to track shipment, to receive payment, to manage orders and to view customer details. To solve the problems faced by various organizations our paper proposes 'Inventory Management Software'- online inventory management software that keeps track of stock levels, helps to manage orders which reduces the trouble they face by using multiple applications. The inventory management software is a useful and mandatory tool which makes use of business intelligence to provide organizations with a complete overview of their inventory just by looking at its dashboard. Various graphs are generated by this software which indicate the stock levels on daily, monthly or weekly basis. It's real-time inventory dashboard to help optimize sales and inventory levels to get critical insights of the business whenever required. Thus, software is used for analytics purpose using various machine learning algorithms to run an efficient business.
\end{abstract}

\section{General Terms}

Marketing, E-commerce Organizations, Inventory, Optimize Sales

\section{Keywords}

Business Intelligence, E-commerce, Inventory Management Software, Machine Learning

\section{INTRODUCTION}

Business intelligence[2] is used by organizations to improve their business in assortments, replenishment, vendors and to update stocks. Business intelligence are the strategies applied for the business to flourish. To track, manage and organize product sales, material purchases and other production processes are the constituents of the inventory management software. Using these businesses reduce the time and efforts previously put into basic tracking, and instead focus on analyzing, finding and reducing inefficiencies in their model. The software is able to forecast the demands[3] of the online marketing industry i.e. ensuring that the products needed for the business to run are always available in the right quantities. This means that there should neither be too much purchased nor too little, but just the right amount required with adequate backup.

\subsection{Benefits of using Business Intelligence:}

1. Business intelligence helps businesses refine an assorted inventory and optimize supply quantities and inventory allocation across stores, by analyzing detailed historical data such as buying patterns and the demographics of customers.

2. Minimize the risk of out-of-stock situations by analyzing safety stock data and inventory and sales information to create accurate forecasts - business intelligence provides the valuable data that a forecasting tool for small business needs.

3. Predict over-stock situations before they become a major problem by drawing on replenishment, sales, and forecasting data.

4. Reduce in the long-term the number of inventory items with a slow turnover or which simply gather dust, by measuring demand and sale levels on a per-location and per-season basis, while taking into account the impact of price changes.

5. Use physical inventory counts to generate inventory verification schedules and thus maintain highly accurate inventory data that can be used to inform the ordering process.

6. Analyze and monitor inventory levels for both finished goods and the materials used to product the finished goods and better understand buyer behavior, in order to improve the cost-effectiveness of the inventory control process.

7. Determine the most time-effective and cost-effective way to get rid of slow-selling inventory by identifying the discounts that have had the most significant impact in the past as well as the locations where specific products have sold best.

\section{LITERATURE SURVEY}

Since India is moving towards digitization wherein we are promoting cashless India, Online marketing has become the upcoming trend where all the business firms are shifting their business online. All online marketing websites are in a race to improve their business. This requires a lot of efforts; in our system we have tried to automate the manual efforts.

It is very essential to analyze the sale of an e-commerce website in order to manage the stocks and also to obtain trends and patterns of sale. In our system we perform analysis on the transactions data then by using various machine learning techniques we will obtains the trends and patterns of products sold. Now after obtaining patterns we can optimize the sales of the system and thereby we can manage the inventory of our system.

Machine Learning[5]: Without being explicitly programmed, it provides the computers with the ability to learn. It is a type of artificial intelligence(AI). Through machine learning we can develop computer programs that can change when exposed to new data.

Machine learning is similar to data mining. Both techniques are similar as they both look for patterns in the data. However 
machine learning uses that data to detect patterns in data and adjust program actions accordingly.

Thus in order to detect patterns of sales of products and to optimize the sales we will use a few machine learning techniques[4] in our system.

\subsection{Logistic Regression}

Logistic regression is a machine learning algorithms for binary classification. It is a simple algorithm that performs very well on a wide range of problems.

Logistic regression uses logistic function. The logistic function is a sigmoidal function, it was developed to describe properties of population growth etc. The graph of logistic function is an S-shaped curve which can take any real values and can map it into a value between 0 and 1 . It can be given as

\section{$1 /\left(1+\mathrm{e}^{\wedge}\right.$-value $)$}

Where e is the base of the natural logarithms.

Below is a plot of the numbers between -5 and 5 transformed into the range 0 and 1 using the logistic function.

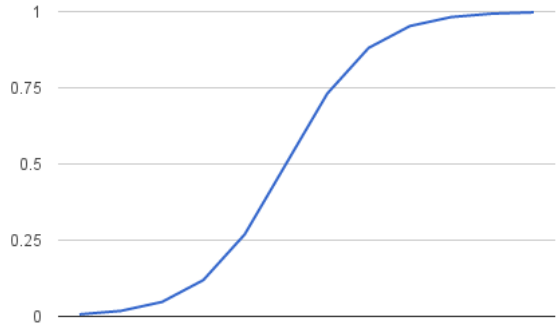

Figure 2. Logistic Function

Logistic regression helps in modeling the probability of a class.For example, if we want to model people's sex as male or female from their height, then the class could be male , hence the logistic regression model can be written as the probability of male given a person's height, or more formally:

\section{$\mathrm{P}(\mathrm{sex}=$ male|height $)$}

Hence in our system we will use logistic regression in order to predict the probability that a product will be sold or not based on the past sales.

\subsection{Market Basket Analysis}

Affinity analysis and association rule learning encompasses a set of analytics techniques which helps at uncovering the associations and connections between specific objects.Of these, "market basket analysis" is a famous example. In a market basket analysis, we look to see the combinations of products sold together.

For example: People, who buy flour and casting sugar, can also buy eggs (in order to prepare a cake).
We use this technique to:

- Inform where to place the content of items on the media sites or in the catalogue of products.

- Help the recommendation engines to recommend products to the customers.

- Implement targeted marketing (e.g. to email customers who bought specific products with other products and to give offers on those products that are likely to be interesting to them.)

\section{EXISTING SYSTEMS}

\subsection{QuickBooks}

This is fast and rapidly growing inventory management system[6] . It gives a real-time insights to stay in control. It gives the complete summary of the business on the home screen itself. It gives a timeline view of all the recent transactions, it gives a graphical view of cash flow and business performance. QuickBooks gives you the benefits of working online.

\section{Disadvantages:}

1. It has a limited reporting and transparency of your business health

2. It gives errors of Double entry and keying

3. It gives limitations with file size and data

4. There is lack of integration in standalone application.

\subsection{Jazva Inventory}

Jazva[7] has a powerful single platform enterprise solution that helps online retailers and wholesalers simplify, synchronize, and automate multichannel selling. It is the backend system. It acts like invisible pieces of the jigsaw that binds everything together and without which an effective multichannel business can not happen. Jazva's inventory softaware helps retailers to sell, scale, and sell more without the need to use multiple softwares to run their business.

\section{Disadvantages:}

1. The report generator in this software is not intuitive.

2. It requires a good understanding of the logic behind the system.

\section{OUR APPROACH}

On studying all the current existing system, we have tried to overcome the disadvantages of the existing system by proposing Inventory Management Software. Our approach in developing our system is shown in the given block diagram (Figure 3). 


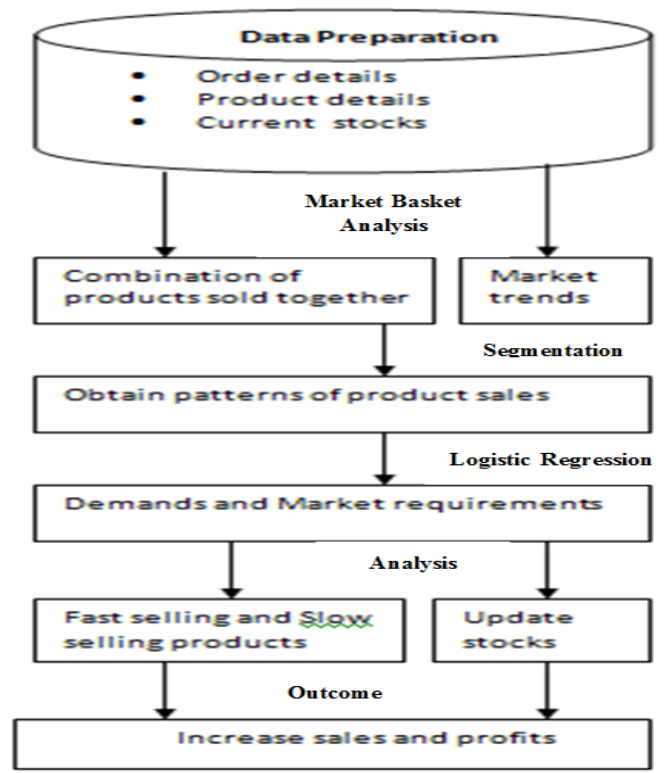

Figure 3. Block diagram of " Inventory management software"

As shown in the (Figure 3) we first prepare our data for use. This data consists of all the sales data i.e order details, product details and the current stocks of each product. Then we apply Market Basket Analysis algorithm on this data. Using this algorithm, we obtain the combination of products sold together and we obtain the current market trends. We now segment the market trends to obtain the patterns of product sales. On applying logistic regression to these patterns, we can forecast the demands of the products and thus obtain the market requirements.

We now analyze the market requirements in order to segregate the data as fast-selling and slow-selling products . Now according to product sales we update the stocks of products. Our main aim is to increase the sales and increase the overall profits.

\section{IMPLEMENTATION}

\subsection{Django framework}

The inventory management software has been developed by using the Django framework it is an open source web application written in python. It helps to develop a web application more faster and easier way. Using Django we can easily create a large or complex databases that can be cconsist of website, also it provide an administrative side in that we can create, update, read and delete the database dynamically.

\subsection{Specifications:}

- Front end: Python 2.7, HTML5, CSS, JavaScript, JQuery

- $\quad$ Back end: PostgreSQL

- Software tools: JetBrains, PyCharm

\subsection{Working of Minimal Inventory Management System:}

As mentioned above the software performs Inventory by keeping a track of the sales of products, orders placed, stock levels and vendor profile details.
The Dashboard (Fig 4.) gives the overview of all the activities performed by the software, which consists of the revenue generated. It also shows the product status with respect to stocks.

In (Fig 5.) a system generated graph is depicted which shows the current daily sales revenue which is increasing or decreasing sales profits.

The stock level graph is shown in (Fig 6.) This displays the current stock levels of each product. It helps the vendor to recognize which product stocks needs to be updated.

The next graph is Completed Tasks (Fig 7) which shows the tasks completed by the vendor which includes the order fulfillment, stock updating etc.

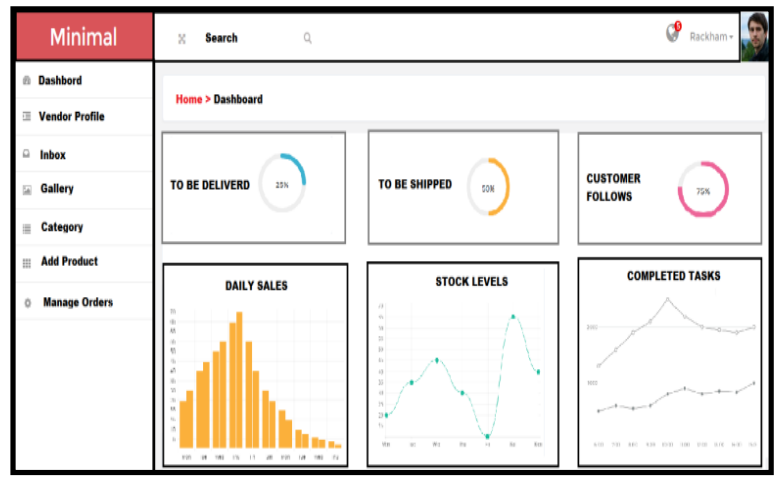

Figure 4. Dashboard

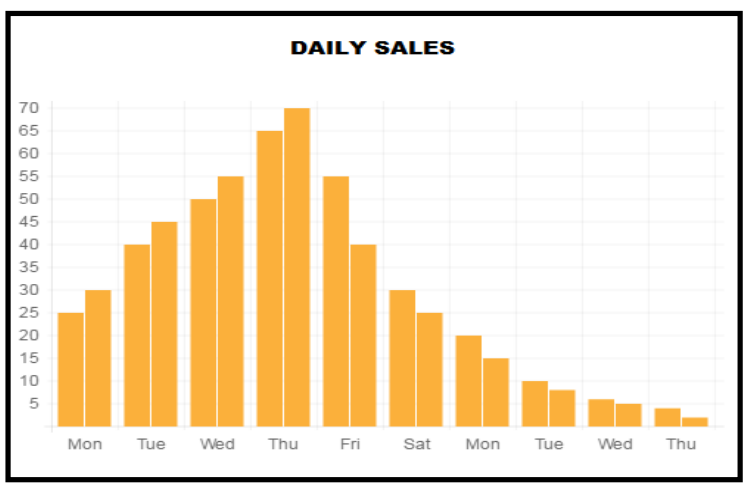

Fig 5. Daily Sales

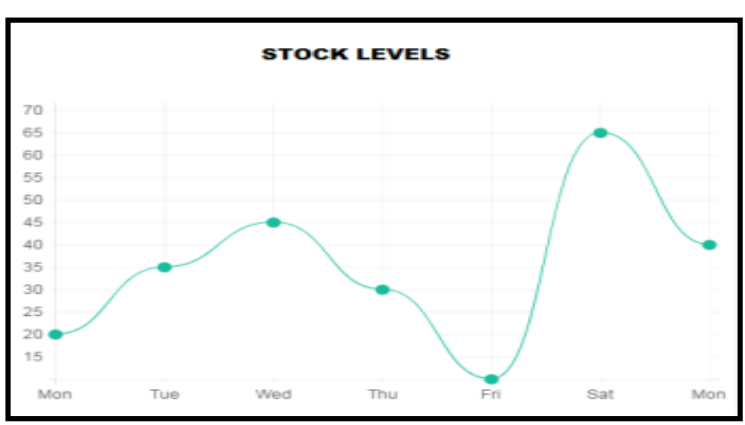

Figure 6. Stock Levels 


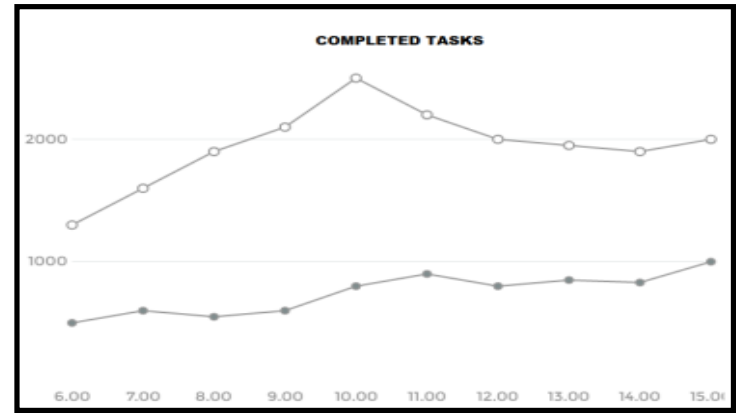

Figure 7. Completed Tasks

\section{CONCLUSION}

Today's world are relying on Business Intelligence applications in that organizations are collect data from internal and external sources, prepare it for analysis, develop and run queries against the data, and create reports, dashboards and provide more informed decisions making strategies. Hence large organizations should tackle BI strategically, because they have valuable data that can tell them about performance, Market strategy, customer behavior, process efficiency and important trends. Also the various machine learning algorithms such as market basket analysis and linear regression are used to track, manage and organize product sales, product purchases and also helps to produce patterns of product sales. As the software is implemented in Django framework, it is robust and efficient to withstand the changing requirements of the online marketing industry. Therefore, software increases the sales and profits of various organizations.

\section{ACKNOWLEDGMENTS}

We would like to express deepest appreciation towards Dr. Mohiuddin Ahmed, Principal MHSSCOE, Mumbai and Dr. Z.A Usmani, H.O.D Computer Department whose invaluable guidance supported us in this project.We are profoundly grateful to Dr. Zainab Pirani for her expert guidance and continuous encouragement throughout to see that this research paper rights its target. At last we must express our sincere heartfelt gratitude to all the staff members of Computer Engineering Department who helped us directly or indirectly during this course of work.

\section{REFERENCES}

[1] W Xu, D.P.Song, Roe M. 2010 Supply chain performance improvement using vendor Management Inventory Strategy, 2010 IEEE International Conference on Industrial Engineering and Engineering Management.

[2] Malek Sarhani, AbdellatifAfia EI. 2014 Intelligent system based support vector regression for Supply chain demand forecasting, IEE Second World Conference on Complex Systems (WCCS).

[3] Fang Tu, Sudipto, Ghoshal Jianhui, Luo Gautam, Biswas Sankaran, Mahadevan Link Jaw, Kelly N. 2007 PHM Integration with Maintenance and Inventory Management System, IEEE Aerospace Conference.

[4] Yoo P.D., M.H. Kim, Jan T. 2005 Machine Learning Techniques And Use of Event Information for Stock Market Prediction on: A Survey and Evaluation, International Conference on Computational Intelligence for Modelling, Control and Automation and International Conference on Intelligent Agents, Web Technologies and Internet Commerce (CIMCA-IAWTIC'06).

[5] Pradip Kumar 2010 Decision tree based demand forecasts for improving inventory Performance, IEEE International Conference on Industrial Engineering And Engineering Management.

[6] https://quickbooks.intuit.com/inventory-management/

[7] https://www.jazva.com/features/inventory-management 